\title{
On the Splitting of Mapping Spaces between Classifying Spaces I
}

\author{
Dedicated to the memory of Professor Shichiro Oka
}

By

\section{Norio IVASE*}

\section{§ 0 . Introduction}

A homomorphism from a product group of simple Lie groups to a simple Lie group cannot be a "crossed" homomorphism, unless the dimension of the source group is less than the one of the target group. This fact is closely related to the fact that the multiplication of a simple Lie group is not abelian and the classifying space is not an $H$-space. In this paper we show that the same statement replacing a Lie group and a homomorphism with a classifying space and a continuous mapping is valid in the case where the target space is a classifying space of a Lie group of rank one. To show this, we give another representation of a theorem of Miller [5].

Main Theorem. Let $G_{1}$ and $G_{2}$ be compact connected Lie groups with finite fundamental groups and $H$ a simple Lie group of rank 1 i.e. $H=S O(3)$ or $S p(1)$. Then the canonical projections $p_{t}$ of $B G_{1} \times B G_{2}$ to the $t$-th factors induce tho weak equivalences

$$
\begin{aligned}
& p_{1}^{*} \cup_{B H} p_{2}^{*}: \operatorname{Map}\left(B\left(G_{1} \times G_{2}\right), B H\right) \simeq{ }_{w} \operatorname{Map}\left(B G_{1}, B H\right) \cup_{B H} \operatorname{Map}\left(B G_{2}, B H\right), \\
& p_{1}^{*} \vee p_{2}^{*}: \operatorname{Map}_{*}\left(B\left(G_{1} \times G_{2}\right), B H\right) \simeq{ }_{w} \operatorname{Map}_{*}\left(B G_{1}, B H\right) \vee \operatorname{Map}_{*}\left(B G_{2}, B H\right),
\end{aligned}
$$

where $\operatorname{Map}(A, B)$ and $\operatorname{Map}_{*}(A, B)$ are the spaces of all mappings from 4 to $B$ which are base point free and base point preserving respectively, $B G$ denotes the classifying space of any group $G, \simeq{ }_{w}$ denotes a weak equivalence and $\cup_{Y}$ means the pushout over $Y$.

Corollary. Let $G_{i}$ be compact connected Lie groups with finite fundainental groups, $i=1, \cdots, r$, and let $H$ bc a simple Lie group of rani ons. Then the canonical projections $p_{t}$ to the $t$-th factors induce the bijection

Communicated by N. Shimada, August 5, 1985. Revised July 4, 1936.

* Department of Mathematics, Kyushu University 33, Fukuoka 812, Japa?. 


$$
\vee_{t} p_{t}^{*}: \pi_{0}\left(\operatorname{Map}_{*}\left(B \prod_{i=1}^{r} G_{i}, B H\right), *\right) \cong \bigvee_{t=1}^{r} \pi_{0}\left(\operatorname{Map}_{*}\left(B G_{t}, B H\right), *\right)
$$

The corollary shows that $B S p(1)$ and $B S O(3)$ do not allow $H$-structures, and moreover, have no binary operations except for the trivial one. And we may give an example to the homotopy set of all self homotopy equivalences, which is suggested by the referee.

Example 4.3. $\operatorname{SHE}\left(\Pi^{n} H P^{\infty}\right) \cong \Sigma_{n}$ the $n$-th symmetric group, where $\operatorname{SHE}(X)$ is the homotopy set of all self homotopy equivalences of a space $X$.

This example is simple and is a contrast to the fact that $\operatorname{SHE}\left(\Pi^{n} C P^{\infty}\right) \cong$ $G L_{n}(\boldsymbol{Z})$ which is an infinite group if $n \geqq 2$. The proof of Main Theorem depends deeply on Lemma 1.1 and Theorem 1.5 which are stated as follows.

Lemma 1.1. Let $G$ be a maximal compact subgroup of a reductive complex Lie group and $Y$ a finite dimensional $C W$-complex. Theu any element of $\pi_{q} \operatorname{Map}_{*}(B G, Y)$ is represented by a phantom mapping for all $q \geqq 0$.

Theorem 1.5. Let $Y$ be simply connected and each $X_{t}$, for $t=1$ and $2, a$ connected $C W$-complex such that $\operatorname{Mav}_{*}\left(X_{t}, \Omega Y\right)$ is weakly contractible. If any mapping $h$ of $X_{1} \times X_{2}$ to $Y$ is homotopic to the mapping which annihilates one of the subspaces $X_{1} \times\{*\}$ and $\{*\} \times X_{2}$, then the natural projections $p_{t}$ of $X_{1} \times X_{2}$ to $X_{t}$ induce the weak equivalences

$$
\begin{aligned}
& p_{1}^{*} \cup_{Y} p_{2}^{*}: \operatorname{Map}\left(X_{1} \times X_{2}, Y\right) \simeq{ }_{w} \operatorname{Map}\left(X_{1}, Y\right) \cup{ }_{Y} \operatorname{Map}\left(X_{2}, Y\right), \\
& p_{1}^{*} \vee p_{2}^{*}: \operatorname{Map}_{*}\left(X_{1} \times X_{2}, Y\right) \simeq{ }_{w} \operatorname{Map}_{*}\left(X_{1}, Y\right) \vee \operatorname{Map}_{*}\left(X_{2}, Y\right) .
\end{aligned}
$$

Note that Lemma 1.1 is already known and stated for the case $q=0 \quad[3]$ which is used in [9], and is another representation of theorems of Miller [4, 5].

The author wishes to express deeply his gratitude to late Professor Shichiro Oka for his heartful encouragement and the usefull discussions with him. He thanks Professors M. Mimura and A. Zabrodsky for their valuable comments, and also the referee for his usefull suggestions and comments.

This paper is organized as follows. We prove Main Theorem dividing into the two cases $H=S p(1)$ and $H=S O(3)$. At first, we prepare the Lemma 1.1 and prove Main Theorem in the case when $H=S p(1)$ using theorem 1.5 and Proposition 1.3 in Section 1 and prove Theorem 1.5 in Section 2. Next, we prove Main Theorem in the case $H=S O(3)$ in Section 3. Finally, we shall give in Section 4 some applications of Main Theorem and show in Appendix that there are some counter-examples if we omit any hypothesis of Main Theorem. 


\section{$\S 1$. Preliminaries}

Throughout this paper, we denote by $S^{q}$ the functor taking $q$-fold reduced suspension and by $\Omega$ the loop space functor. We at first generalize slightly the theorem of Miller [5], using the theory of phantom mappings due to Zabrodsky [8].

Lemma 1.1. Let $G$ be a maximal compact subgroup of a reductive complex Lie group and $Y$ a finite dimensional $C W$-complex. Then any element of $\pi_{q} \operatorname{Map}_{*}(B G, Y)$ is represented by a phantom mapping for all $q \geqq 0$.

Proof. By the theorem of E. M. Friedlander and G. Mislin [3], for each prime $p$, we can take a locally finite group $\pi$ and a mapping $\phi: B \pi \rightarrow B G$ which induces a $\mathbb{Z} / p \mathbb{Z}$-cohomology isomorphism. For any mapping $f$ of $\operatorname{Map}_{*}\left(S^{q}(B G), Y\right)$, $f\left(S^{q}(\phi)\right)$ belongs to $\operatorname{Map}_{*}\left(S^{q}(B \pi), Y\right)$ which is weakly contractible by H. Miller [5]. Therefore, $f\left(S^{q}(\phi)\right)$ is null-homotopic. We can take the Sullivan completion of $Y$ provided that $Y$ is simply connected. We at first assume that $Y$ is simply connected and take the Sullivan $p$-completion $\hat{e_{p}}: Y^{\gamma} \rightarrow Y \hat{p}$. Then we have

$$
\hat{e_{p}} f\left(S^{q}(\phi)\right) \simeq^{*},
$$

in $\operatorname{Map}_{*}\left(S^{q}(B \pi), Y_{p}^{\hat{p}}\right)$. On the other hand, since $H^{*}\left(S^{q}(\phi) ; Z \mathbb{Z} / p \mathbb{Z}\right)$ is an isomorphism, $\hat{e_{p} f}$ is null-homotopic. Hence the composition of $f$ with the Sullivan completion mapping $e^{\wedge}: Y \rightarrow Y^{\wedge}$ is null-homotopic. This implies that $f$ is a phantom mapping. When $Y$ is not simply connected, we take the universal covering space $\bar{Y}$ of $Y$. Then $\bar{Y}$ is a simply connected, finite dimensional complex and this lemma holds for $\bar{Y}$. $f$ induces the trivial homomorphism between fundamental groups. If it were not so, then there should exist an element $g$ of $G$ such that the composition of $f$ with the mapping $B\langle g\rangle \rightarrow B G$ induced from the inclusion is non-trivial. This contradicts to the theorem of H. Miller [5]. Hence, all mappings from $S^{q}(B G)$ to $Y$ induce trivial homomorphisms between their fundamental groups, $q \geqq 0$, and the covering projection induces a following continuous bijection:

$$
\operatorname{Map}_{*}\left(S^{q}(B G), \bar{Y}\right) \cong \operatorname{Map}_{*}\left(S^{q}(B G), Y^{*}\right),
$$

which maps a phantom mapping to a phantom mapping. and moreover, is a homeomorphism, since a covering projection is an open mappirg. This completes the proof of the lemma.

Using this, we get

Proposition 1.2. Let $G$ be a compact connected Lie group and $Y$ a finite dimensional $C W$-complex with finite homotopy groups in all dintinsions except for 
2 and 3. Then the space $\operatorname{Map}_{*}(B G, Y)$ is weakly contractible.

Proof. Recall that the Chevalley's complexification of the compact connected Lie group $G$ is a complex reductive Lie group whose maximal compact subgroup is conjugate with $G([1])$. By Lemma 1.1, it is sufficient to prove that the space of all phantom mappings from $B G$ to $Y$ is weakly contractible. According to A. Zabrodsky [8], the $q$-th homotopy group of the space of all phantom mappings from a $C W$-complex $X$ of finite type with a finite fundamental group to a simply connected $C W$-complex $Z$ of finite type is a quotient group of $\Pi_{n>0} \operatorname{Ext}\left(\bar{H}_{n}(X ; \boldsymbol{Q}), \pi_{n+1}(Z) /\right.$ torsion $)$. For the dimensional reasons, this must be 0 , and the proof of this proposition is completed.

We prepare a cohomological information as follows.

Proposition 1.3. Let $G_{1}$ and $G_{2}$ be connected compact Lie groups with finite fundamental groups, $S p(1)$ the symplectic group of rank 1 and $j_{t}: B G_{t} \rightarrow B G_{1} \times B G_{2}$ be the canonical inclusion into the $t$-th factor $t=1,2$. Then for any mapping $h: B G_{1} \times B G_{2} \rightarrow B S p(1)$, we have $H^{*}\left(h j_{1} ; \boldsymbol{Z}\right)=0$ or $H^{*}\left(h j_{2} ; \boldsymbol{Z}\right)=0$.

Proof. Since $H^{*}(B S p(1) ; \boldsymbol{Z}) \cong \boldsymbol{Z}\left[w_{4}\right]$, it is sufficient to show that $H^{*}\left(h j_{t} ; \boldsymbol{Z}\right)\left(w_{4}\right)$ $=0$, for $t=1$ or 2 . Let $q_{t}: \bar{G}_{t} \rightarrow G_{t}$ be a universal covering of $G_{t} . H^{4}\left(B G_{t} ; \boldsymbol{Z}\right)$ is a free abelian group of finite rank $r_{t}, t=1$ or 2 , over $\boldsymbol{Z}$ and $H^{4}\left(B q_{t} ; \boldsymbol{Z}\right)$ is injective by the dimensional reasons in the Serre spectral sequence for $B \bar{G}_{t} \rightarrow$ $B G_{t} \rightarrow K\left(\pi_{1}\left(G_{t}\right), 2\right)$. Since $\bar{G}_{t}$ is simply connected and semi-simple, there is an inclusion homomorphism;

$$
i_{t}:(S p(1))^{r} \longrightarrow \bar{G}_{t}
$$

such that $H^{4}\left(B i_{t} ; \boldsymbol{Z}\right)$ is an isomorphism. If we assume $H^{*}\left(h j_{1} ; \boldsymbol{Z}\right)\left(w_{4}\right)=\sum_{\imath=1}^{r_{1}} a_{\imath} u_{i}$ $\neq 0$ and $H^{*}\left(h j_{2} ; \boldsymbol{Z}\right)\left(w_{4}\right)=\sum_{\jmath=1}^{r_{2}} b_{j} v_{\jmath} \neq 0$, then we may assume $a_{1} \neq 0, \quad b_{1} \neq 0$ and $\bar{H}^{*}\left(h B\left(q_{t} i_{t} i n_{t}\right) ; \boldsymbol{Z}\right)\left(w_{4}\right) \neq 0$, where $i n_{t}: S p(1) \rightarrow(S p(1))^{r_{t}}$ is the canonical inclusion into the first coordinate. Let $k$ be the following mapping

$$
h\left(B\left(q_{1} i_{1} i n_{1}\right) \times B\left(q_{2} i_{2} i n_{2}\right)\right): B S p(1) \times B S p(1) \longrightarrow B S p(1),
$$

then we get $\bar{H}^{*}(k ; \boldsymbol{Z})\left(w_{4}\right)=a w_{4} \times 1+1 \times b w_{4}, a b \neq 0$.

Take an odd prime $p$ to be mutually prime with $a b$, and then $a b \neq 0$ in $\boldsymbol{Z} / p \boldsymbol{Z}$ and it contradicts to the commutativity of $\bar{H}^{*}(k ; \boldsymbol{Z} / p \boldsymbol{Z})$ with the Steenrod $p$-th power operation $P^{1}$.

Actually, $\bar{H}^{*}(k ; \boldsymbol{Z} / p \boldsymbol{Z})\left(P^{1} w_{4}\right)=2 \bar{H}^{*}(k ; \boldsymbol{Z} / p \boldsymbol{Z})\left(w_{4}^{(p+1) / 2}\right)=2\left(a w_{4} \times 1+1 \times b w_{4}\right)^{(p+1) / 2}$ $=2 a^{(p+1) / 2}\left(w_{4}\right)^{(p+1) / 2} \times 1+1 \times 2 b^{(p+1) / 2}\left(w_{4}\right)^{(p+1)}+2 a^{(p-1) / 2} b\left(w_{4}\right)^{(p-1) / 2} \times w_{4}+\cdots$, while $P^{1} \bar{H}^{*}(k ; \boldsymbol{Z} / p \boldsymbol{Z})\left(w_{4}\right)=2 a\left(w_{4}\right)^{(p+1) / 2} \times 1+1 \times 2 b\left(w_{4}\right)^{(p+1)}$.

It is a contradiction. This completes the proof of this proposition.

Remark 1.4. Combining this with Zabrodsky's Theorem ([9], Theorem 2), 
the conclusion of the above lemma can be translated to the foilowing form:

$$
h \mid\{*\} \times B G_{2} \simeq * \text { or } h \mid B G_{1} \times\{*\} \simeq * \text {. }
$$

We state here the following theorem which will be proved in Section 2 .

Theorem 1.5. Let $Y$ be simply connected and $X_{1}$ and $Y_{2}$ such connected $C W$ complexes that $\operatorname{Map}_{*}\left(X_{t}, Y\right)$ is weakly contractible for $t=1,2$. If any mapping $h$ of $X_{1} \times X_{2}$ to $Y$ is homotopic to the mapping which annihila:cs one of the subspaces $X_{1} \times\left\{{ }^{*}\right\}$ and $\left\{{ }^{*}\right\} \times X_{2}$. Then there exist weak equivalences

$$
\begin{aligned}
& \operatorname{Map}\left(X_{1} \times X_{2}, Y\right) \simeq{ }_{w} \operatorname{Map}\left(X_{1}, Y\right) \cup \cup_{Y} \operatorname{Map}\left(X_{2}, Y\right), \\
& \operatorname{Map}_{*}\left(X_{1} \times X_{2}, Y\right) \simeq{ }_{w} \operatorname{Map}_{*}\left(X_{1}, Y\right) \vee \operatorname{Map}_{*}\left(X_{2}, Y\right) .
\end{aligned}
$$

This together with Proposition 1.2, Proposition 1.3 and Remark 1.4 implies Main theorem in the case when $H=S p(1)$.

\section{$\S 2$. Proof of Theorem 1.5}

Since $Y$ is simply connected, we have isomorphisms

$$
\begin{gathered}
\pi_{0}\left(\operatorname{Map}_{*}\left(X_{1} \times X_{2}, Y\right), *\right) \cong \pi_{0}\left(\operatorname{Map}\left(X_{1} \times X_{2}, Y\right), *\right), \\
\pi_{0}\left(\operatorname{Map}_{*}\left(X_{t}, Y\right), *\right) \cong \pi_{0}\left(\operatorname{Map}\left(X_{t}, Y\right), *\right), \\
\operatorname{Map}\left(X_{1} \times X_{2}, Y\right) \cong \operatorname{Map}\left(X_{1}, \operatorname{Map}\left(X_{2}, Y\right)\right) \\
=\Perp_{a} \operatorname{Map}\left(X_{1}, C_{\alpha}\left(X_{2}, Y\right)\right),
\end{gathered}
$$

where $C_{a}(X, Z)$ is the connected component of $\alpha$ in $\operatorname{Map}(X, Z)$ and $\alpha$ runs over all connected components. We similarly denote the connected component of $\alpha$ in $\operatorname{Map}_{*}(X, Z)$ by $C_{a}(X, Z)$. Note that $C_{0}\left(X_{t}, Y^{-}\right)$is weakly contractible by the assumption. And by Zabrodsky's Lemma ([9], Lemma 1.5), the evaluation mapping $e v_{t}: C_{0}\left(X_{t}, Y\right) \rightarrow Y$ is a weak equivalence. Hence we can see that the composition mapping $\left(e v_{2}\right)_{\#}: \operatorname{Map}\left(X_{1}, C_{0}\left(X_{2}, Y\right)\right) \rightarrow \operatorname{Map}\left(X_{1}, Y\right)$ is also a weak equivalence. Assume $\alpha \neq 0$ and take the representative mapping $f$ of $\alpha$. For any mapping $h$ of $\operatorname{Map}\left(X_{1}, C_{\alpha}^{\hat{\alpha}}\left(X_{2}, Y\right)\right)$, the diagram (2.1) is homotopy commutative by the assumption. On the other hand, a mapping which makes the diagram (2.1) homotopy commutative belongs to $\operatorname{Map}\left(Y_{1}, C_{a}\left(X_{2}, Y\right)\right)$;

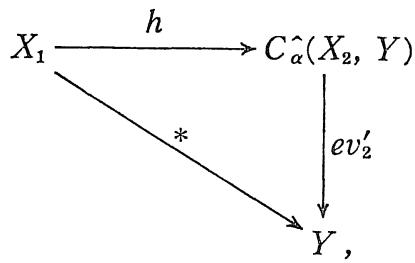


where $e v_{t}^{\prime}: C_{a}^{\wedge}\left(X_{t}, Y\right) \rightarrow Y$ is an evaluation mapping. By double adjoining this mapping $h$, the above homotopy commutative diagram is equivalent to the following one.

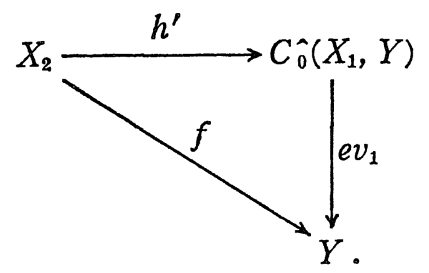

Let us recall that both $e v_{1}$ and the mapping of composing with $e v_{1}$ are weak equivalences. Hence the last term of

$$
\begin{aligned}
\operatorname{Map}\left(X_{1}, C_{a}^{\wedge}\left(X_{2}, Y\right)\right) & =\left(e v_{2}^{\prime}\right)_{\#}^{-1}\left(C_{0}^{\hat{0}}\left(X_{1}, Y\right)\right) \\
& =\left(e v_{1}^{\prime}\right)_{\#}^{-1}\left(C_{a}^{\wedge}\left(X_{2}, Y\right)\right)
\end{aligned}
$$

is weakly homotopic to $C_{a}^{\wedge}\left(X_{2}, Y\right)$. Finally, we obtain that

$$
\operatorname{Map}\left(X_{1} \times X_{2}, Y\right) \simeq{ }_{w} \operatorname{Map}\left(X_{1}, Y\right) \cup_{Y} \operatorname{Map}\left(X_{2}, Y\right),
$$

where we regard the mapping $\operatorname{space} \operatorname{Map}\left(X_{t}, Y\right)$ as $\left(\Perp_{\alpha \neq 0} C_{\alpha}^{\wedge}\left(X_{t}, Y\right)\right) \Perp C_{0}^{\hat{0}}\left(X_{t}, Y\right)$ with $C_{0}\left(X_{t}, Y\right) \cong{ }_{w} Y$.

By the commutativity of the following diagram, the mapping in the upper sequence is also a weak equivalence:

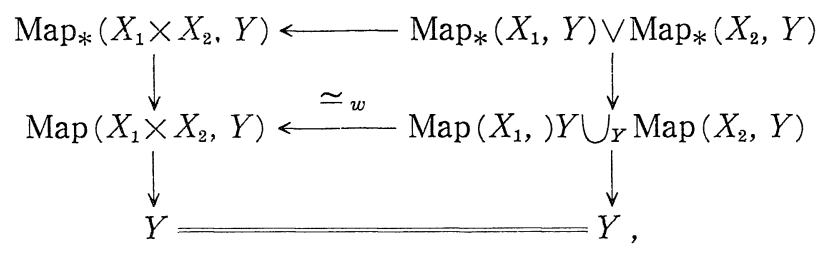

where the mappings in the upper and middle sequences are induced by the canonical projections $p_{t}: X_{1} \times X_{2} \rightarrow X_{t}(t=1,2)$, and we regard the mapping space $\operatorname{Map}_{*}\left(X_{t}, Y\right)$ as $\left(\Perp_{a \neq 0} C_{\alpha}\left(X_{t}, Y\right)\right) \Perp C_{0}\left(X_{t}, Y\right)$ with $C_{0}\left(X_{t}, Y\right) \cong_{w} *$.

\section{$\S 3$. Proof of Main Theorem for $H=S O(3)$}

In this section, to extend Main Theorem for the case when $H=S O(3)$, we use the propositions of Section 2 together with the following propositions.

Proposition 3.1. Let $G$ be a connected compact lie groups with finite fundamental group, $p: \bar{G} \rightarrow G$ the universal covering, and $f$ an arbitrary mapping from $B G$ to $B S O(3)$, Then $f B p \simeq *$ implies $f \simeq *$. 
Proof. Consider the following fibration;

$$
B \bar{G} \stackrel{B p}{\longrightarrow} B G \stackrel{\lambda}{\longrightarrow} K\left(\pi_{1}(G), 2\right) .
$$

Since $\pi_{1}(G)$ is a finite group, $\bar{G}$ is a compact connected Lie group and $\pi_{q} C_{0}(B \bar{G}, B S O(3))=0$ by Proposition 1.2 with $Y=\Omega B S O(3)=S O(3)$. Again by Zabrodsky's Lemma ([9], Lemma 1.5), there exists a mapping $\bar{f}: K\left(\pi_{1}(G), 2\right) \rightarrow$ $B S O(3)$ such that $f \simeq \bar{f} \lambda$. On the other hand, $\pi_{m} \operatorname{Map}_{*}\left(K\left(\pi_{1}(G), 2\right), B S O(3)\right)=$ $\Pi_{n>0} \operatorname{Ext}\left(\bar{H}_{*}\left(K\left(\pi_{1}(G), 2\right) ; Q\right), \pi_{n+m+1}(B S O(3)) /\right.$ torsion $)=0$ by Zabrodsky's Theorems ([8], Theorem 2.1, 2.1.4, Theorem $\left.4.1(C)_{1}\right)$ with the fact that $\pi_{1}(G)$ is finite. So, $\bar{f} \simeq *$. This implies the proposition.

Now, we can prove for $H=S O(3)$ the following proposition instead of Proposition 1.3 and Remark 1.4 .

Proposition 3.2. Let $G_{1}$ and $G_{2}$ be compact connected Lie groups with finite fundamental groups and $j_{t}: B G_{t} \rightarrow B G_{1} \times B G_{2}$ the canonical inclusion into the $t$-th factor, $t=1,2$. Then for any mapping $h: B G_{1} \times B G_{2} \rightarrow B S O(3), h j_{1} \simeq *$ or $h j_{2} \simeq *$.

Proof. Let $p_{t}: \bar{G}_{t} \rightarrow G_{t}$ and $p: S p(1) \rightarrow S O(3)$ be the universal coverings. Then we can take a lift $\bar{h}$ of $h$ such that the following diagram commutes:

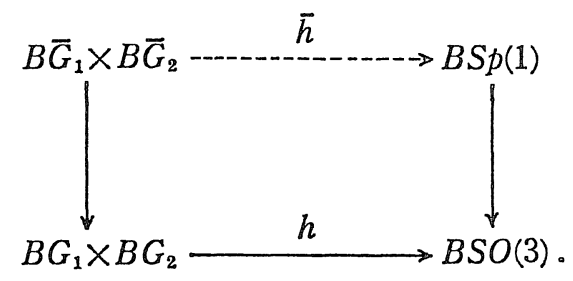

By Proposition 1.3 and Remark 1.4, we have $\bar{h} \mid B \bar{G}_{1} \times\{*\} \simeq *$ or $\bar{h} \mid\{*\} \times B \bar{G}_{2} \simeq *$ 。 Therefore we have $h \mid B G_{1} \times\{*\} \simeq *$ or $h \mid\{*\} \times B G_{2} \simeq *$ by Proposition 3.1. This implies the proposition.

Main Theorem for the case when $H=S O(3)$ is also obtained by Theorem 1.5 together with Proposition 1.2 and 3.2 .

\section{$\S 4$. Applications}

In this section, we determine the homotopy sets and of some mapping spaces between product spaces of $H P^{\infty}$ 's. We will here abbreviate $\pi_{0}\left(\operatorname{Map}_{*}(X, Y){ }^{*}\right)$ by $[X, Y]$, and we denote by $\operatorname{SHE}(X)$ the homotopy set of all self homotopy equivalences.

At first, by Corollary, we have the following example. 
Example 4.1. (1) $\operatorname{Map}\left(\Pi^{n} H P^{\infty}, H P^{\infty}\right)$ is weakly equivalent to $\operatorname{Map}\left(H P^{\infty}, H P^{\infty}\right)$ $\bigcup_{H P^{\infty}} \cdots \bigcup_{H P^{\infty}} \operatorname{Map}\left(H P^{\infty}, H P^{\infty}\right)$ (push-out of $n$ copies).

(2) $\operatorname{Map}_{*}\left(\Pi^{n} H P^{\infty}, H P^{\infty}\right)$ is weakly equivalent to $\operatorname{Map}_{*}\left(H P^{\infty}, H P^{\infty}\right) \vee \cdots \vee$ $\operatorname{Map}_{*}\left(H P^{\infty}, H P^{\infty}\right)^{* 1)}$ (wedge-sum of $n$ copies).

This example shows that $H P^{\infty}=B S p(1)$ does not allow $H$-structure, and moreover, does not have binary operations except for the trivial one. In contrast, $B U(1)$ and $B O(1)$ are $H$-spaces.

Proposition 4.2. The homotopy sat $\left[\Pi^{h} H P^{\infty}, \Pi^{k} H P^{\infty}\right]$ is classified by the degrees of mappings and is isomorphic with $M(k, h ; A)$, where $M(k, h ; A)$ denotes the set of all $(k, h)$-matrices whose entries in each arrow are all zero without one entry and are all belonging to $A=\left\{0, j^{2} ; j\right.$ is an odd number $\}$.

Proof. Let us recall that the degree of a self mapping of $H P^{\infty}$ is in $A$ ([2]). Define the degree $\operatorname{deg}(f)$ of a mapping $f: \Pi^{h} H P^{\infty} \rightarrow H P^{\infty}$ by the vector $\left(a^{1}, \cdots, a^{h}\right)$ of integers, whose $j$-th entry $a^{\jmath}=\operatorname{deg}\left(f^{\jmath}\right)$ is the degree of the restriction $f^{\jmath}$ of $f$ to the $j$-th factor of $\Pi^{h} H P^{\infty}$. Then the entries of $\operatorname{deg}(f)$ are all zero without one entry and are belonging to $A$, since $\left[\Pi^{n} H P^{\infty}, H P^{\infty}\right]$ must be the pointed sum of the $h$ copies of $\left[H P^{\infty}, H P^{\infty}\right]$ by Corollary. Let us define the mapping $D$ of $\left[\Pi^{h} H P^{\infty}, \Pi^{k} H P^{\infty}\right] \cong \Pi^{k}\left[\Pi^{h} H P^{\infty}, H P^{\infty}\right]$ to $M(k, h ; A)$ by

$$
D(f)=\left(\begin{array}{c}
\operatorname{deg}\left(f_{1}\right) \\
\vdots \\
\operatorname{deg}\left(f_{k}\right)
\end{array}\right),
$$

where $f=f_{1} \cdots f_{k}$ and $f_{i}: \Pi^{n} H P^{\infty} \rightarrow H P^{\infty}$. On the other hand, D. Sullivan constructed self mapping of $H P^{\infty}$ of any given odd square degree ([7]), and recently, G. Mislin shows that a self mapping of $H P^{\infty}$ is classified by its degree (see [6], Classification Theorem). Therefore $D$ is a bijection. This implies the proposition.

This proposition implies that the monoid $\left[\Pi^{n} H P^{\infty}, \Pi^{n} H P^{\infty}\right]$ can be regarded as the matrix monoid $M(n, n ; A)$. Hence we obtain

Example 4.3. $\operatorname{SHE}\left(\Pi^{n} H P^{\infty}\right) \cong \Sigma_{n}$ the symmetric group.

\section{Appendix}

The Main Theorem has counter examples if we omit the assumption for $H$ or $G_{t}$ 's.

Counter Examples. 1) Let $H=S p(2), G_{1}=G_{2}=S p(1)$ and $f$ the inclusion of

${ }^{* 1)}$ Recently, W. G. Dwyer shows that every non-trivial component of $\operatorname{Map}_{*}\left(H P^{\infty}, H P^{\infty}\right)$ is weakly equivalent to the completion of $S O(3)$. 
$G_{1} \times G_{2}$ into $\operatorname{Sp}(2)$ which maps $(u, v)$ to the diagonal matrix $u \oplus v$. Then $f$ is a homomorphism with $f \mid G_{1} \times\{e\}$ and $f \mid\{e\} \times G_{2}$ being non-trivial homomorphism, and both of the restrictions to $B G_{1} \times\{e\}$ and $\{e\} \times B G_{2}$ of $B f$ are non-trivial.

2) Let $G_{1}$ and $G_{2}$ be $S O(3)$, and let $H$ be $U(1)$. Put $f: B G_{1} \times B G_{2} \rightarrow B U(1)$ be a representing mapping of $u_{1} \times 1+1 \times u_{2}$ in $H^{2}\left(B G_{1} \times B G_{2} ; \mathbb{Z}\right)$ where $u_{t}$ is a generator of $H^{2}\left(B G_{t} ; \boldsymbol{Z}\right) \cong \boldsymbol{Z} / 2 \boldsymbol{Z}$. Then $f$ has nontrivial restrictions to the factors, because they are just the representing mappings of non-zero elements $u_{1}, u_{2}$.

3) Let $G_{1}$ and $G_{2}$ be non-trivial tori $U(1)^{n}$ and $U(1)^{m}$. (for $k=n$ or $m$, $\pi_{1}\left(\left(U(1)^{k}\right)=Z^{k}\right.$ is an infinite group!) Let $H$ be $S O(3)$ or $S p(1), i: U(1) \rightarrow H$ the injection of the maximal torus, and $m: B G_{1} \times B G_{2} \rightarrow B U(1)$ the multiplication of the $H$-space $B U(1)$. Put $f: B G_{1} \times B G_{2} \rightarrow B H$ be $(B i) m\left(B q_{1} \times B q_{2}\right)$, where $q_{t}: G_{t} \rightarrow$ $U(1)$ are non-trivial projections to some coordinates. Then $f$ has restrictions to the factors which can be regarded as the non-trivial mappings $(B i)\left(B q_{t}\right)$ for $t=1$, 2. (This example is pointed out by the referee.)

\section{References}

[1] Chevalley, C., Theory of Lie groups I, Princeton University Press, 1946.

[2] Feder, S. and Gitler, S., Mappings of quaternionic projective spaces, Bol. de la Soc. Mat. Mex., 18 (1973), 33-37.

[3] Friedlander, E.M. and Mislin, G., Cohomology of classifying spaces of comlex Lie groups and related discrete groups, Comment. Math. Helv., 59 (1984), 347-361.

[4] Miller, H., Massey-Peterson towers and maps from classifying spaces, Lecture Notes in Mathematics, No. 1051 (Springer Verlag, Berlin-Heiderberg-New York, 1983).

[5] - The Sullivan conjecture, Bull. of Amer. Math. Soc., Vol.9, No. 1 (1983), 75-78.

[6] Mislin, G., The homotopy classification of self-maps of infinite quaternionic projective space, preprint.

[7] Sullivan, D., Genetics in homotopy theory and the Adams conjecture, Ann. of Math., 100 (1974), 1-79.

[8] Zabrodsky, A., On phantom maps and a theorem of H. Miller, to appear.

[9] — On maps between classifying spaces, to appear. 
